\title{
A modification of the convergence conditions for Picard's iteration
}

\author{
J. A. EZQUERRO and M. A. HERNÁNDEZ \\ University of La Rioja, Department of Mathematics and Computation \\ C. Luis de Ulloa s/n. 26004 Logroño, Spain \\ E-mail: jezquer@dmc.unirioja.es / mahernan@dmc.unirioja.es
}

\begin{abstract}
The convergence of the method of successive approximations is usually studied by the fixed point theorem. An alternative to this theorem is given in this work, where a contraction mapping is not necessary. An application to nonlinear integral equations of Fredholm type and second kind is also presented.
\end{abstract}

Mathematical subject classification: $45 \mathrm{G} 10,47 \mathrm{H} 17,65 \mathrm{~J} 15$.

Key words: nonlinear equations in Banach spaces, Picard's iteration, semilocal convergence.

The research reported herein was sponsored in part by the Ministry of Science and Technology (BFM 2002-00222), the University of La Rioja (API-03/15) and the Government of La Rioja (ACPI 2003/2004).

\section{Introduction}

Solving nonlinear equations of the form $F(x)=0$, where $F: \Omega \subseteq X \rightarrow X$ is an operator defined on an open convex domain $\Omega$ of a Banach space $X$ with values in $X$, is one of the most known problems in mathematics. Undoubtedly, Newton's method ([2], [6], [8])

$$
x_{n+1}=x_{n}-\left[F^{\prime}\left(x_{n}\right)\right]^{-1} F\left(x_{n}\right), \quad n=0,1,2, \ldots
$$

is one of the most best-known methods for solving these equations. However, the application of the Newton method requires the existence of the operator \#585/03. Received: 07/VII/03. Accepted: 17/III/04. 
$\left[F^{\prime}(x)\right]^{-1}$ in each step and this is not always easy to do. Then, if we think in iterative methods where the operator $F^{\prime}(x)$ does not appear, the first one in that one can think is the method of successive approximations [7]

$$
x_{n+1}=G\left(x_{n}\right)=x_{n}-F\left(x_{n}\right), \quad n=0,1,2, \ldots
$$

which is also known as Picard's iteration [10]. According to the fixed point theorem [9], the operator $G$ must be a contraction so that method (1) is convergent. The aim of this work is centered in modifying this condition, since the number of equations that can be solved by the direct application of (1) is limited. For example, if we consider the nonlinear integral equation ([5]):

$$
x(s)=s+\frac{1}{20} \int_{0}^{1} K(s, t) x(t)^{5} d t, \quad 0 \leq s \leq 1,
$$

for $x \in C[0,1]$, where $K(s, t)$ is the Green function

$$
K(s, t)= \begin{cases}(1-s) t, & t \leq s, \\ (1-t) s, & s \leq t,\end{cases}
$$

then the operator

$$
G(x)(s)=s+\frac{1}{20} \int_{0}^{1} K(s, t) x(t)^{5} d t, \quad 0 \leq s \leq 1,
$$

corresponding to equation (2) and appearing in (1) is not a contraction in the space $C[0,1]$ with the max-norm. In this case, the fixed point theorem ([9]) does not guarantee the convergence of (1) to solve (2). In order to approximate solutions of the equation $F(x)=0$ in situations of this type, we study the semilocal convergence of (1) under an alternating condition, where the operator $F$ satisfies the following condition:

$$
\left\|F^{\prime}(x)-I_{X}\right\| \leq \omega(\|x\|)
$$

where $\omega: \mathbb{R}_{+} \rightarrow \mathbb{R}_{+}$is a non-decreasing function.

The study presented here belongs to the class of unbounded generalized contraction results, where the main idea is to generalize the fixed point theorem using nonlinear majorant function in the contraction inequality, see [1]. In this 
way, condition (4) is connected with the definition of contractor ([1]), since if $\omega \equiv$ constant $<1$, then the identity operator $I_{X}$ is a contractor, so that the fixed point theorem can be generalized.

Note that the convergence analysis given in this paper is semilocal. We replace the strong condition, where the operator involved must be a contraction, which appears in the fixed point theorem and guarantees the global convergence, by condition (4), along with some conditions on the initial iterate $x_{0}$ of the method.

We also obtain conclusions about the existence of solutions of the equation $F(x)=0$, along with the domain of location of them, without finding the solutions themselves. This is sometimes more important than the actual knowledge of a solution.

We observe that, under condition (4), we can guarantee the convergence of method (1) to a solution of (2), which is approximated by Picard's iteration. Finally, an application to nonlinear integral equations of Fredholm type and second kind is given.

\section{Semilocal convergence}

We analyse, under certain conditions for the operator $F$ and the starting point $x_{0}$, the convergence of (1) to a solution of the equaiton $F(x)=0$. Let us suppose $x_{0} \in \Omega$ and the following conditions:

$\left(\mathbf{C}_{1}\right)\left\|x_{1}-x_{0}\right\| \leq \eta$.

$\left(\mathbf{C}_{2}\right)\left\|F^{\prime}(x)-I_{X}\right\| \leq \omega(\|x\|), x \in \Omega$, and $\omega: \mathbb{R}_{+} \rightarrow \mathbb{R}_{+}$is a non-decreasing function.

$\left(\mathbf{C}_{3}\right)$ Assume that $R$ exists, the smallest positive root of the equation

$$
\left(1-\omega\left(\left\|x_{0}\right\|+t\right)\right) t-\eta=0 .
$$

$\left(\mathbf{C}_{4}\right) \overline{B\left(x_{0}, R\right)} \subset \Omega$.

Next, two technical lemmas are given. 
Lemma 2.1. From all $\left(\boldsymbol{C}_{1}\right)-\left(\boldsymbol{C}_{4}\right)$, it follows

$$
\sum_{i=0}^{n}\left(\omega\left(\left\|x_{0}\right\|+R\right)^{i}\right) \eta<\frac{\eta}{1-\omega\left(\left\|x_{0}\right\|+R\right)}=R .
$$

Lemma 2.2. Taking into account all $\left(\boldsymbol{C}_{1}\right)-\left(\boldsymbol{C}_{4}\right)$, we have

a) $\left\|x_{n+1}-x_{n}\right\|<\omega\left(\left\|x_{0}\right\|+R\right)\left\|x_{n}-x_{n-1}\right\|, n \geq 1$,

b) $\left\|x_{n+1}-x_{0}\right\|<\left[1+\sum_{i=0}^{n} \omega\left(\left\|x_{0}\right\|+R\right)^{i}\right] \eta<R, n \geq 1$.

Proof. Firstly, from $\left(\mathbf{C}_{1}\right)$ and $\left(\mathbf{C}_{3}\right)$, we have $x_{1} \in B\left(x_{0}, R\right)$, since $\left\|x_{1}-x_{0}\right\| \leq$ $\eta<R$. Now, taking into account Taylor's formula and (1), we have

$$
F\left(x_{1}\right)=\int_{0}^{1}\left(F^{\prime}\left(x_{0}+t\left(x_{1}-x_{0}\right)\right)-I_{X}\right) d t\left(x_{1}-x_{0}\right) .
$$

Hence

$$
\begin{aligned}
\left\|F\left(x_{1}\right)\right\| & \leq\left(\int_{0}^{1}\left\|F^{\prime}\left(x_{0}+t\left(x_{1}-x_{0}\right)\right)-I_{X}\right\| d t\right)\left\|x_{1}-x_{0}\right\| \\
& \leq \omega\left(\left\|x_{0}\right\|+R\right)\left\|x_{1}-x_{0}\right\|,
\end{aligned}
$$

since $x_{0}+t\left(x_{1}-x_{0}\right) \in B\left(x_{0}, R\right)$ and $\omega$ is a non-decreasing function. Thus

$$
\left\|x_{2}-x_{1}\right\| \leq \omega\left(\left\|x_{0}\right\|+R\right)\left\|x_{1}-x_{0}\right\|
$$

and, by lemma 2.1,

$$
\left\|x_{2}-x_{0}\right\| \leq\left\|x_{2}-x_{1}\right\|+\left\|x_{1}-x_{0}\right\|<\left(1+\omega\left(\left\|x_{0}\right\|+R\right)\right) \eta<R .
$$

Consequently, $x_{2} \in B\left(x_{0}, R\right)$.

Next, we suppose that a) and b) hold for every $n=1,2, \ldots, i-1$. Then, using again Taylor's formula and (1), we have

$$
\begin{aligned}
\left\|F\left(x_{i}\right)\right\| & \leq \int_{0}^{1}\left\|F^{\prime}\left(x_{i-1}+t\left(x_{i}-x_{i-1}\right)\right)-I_{X}\right\| d t\left\|x_{i}-x_{i-1}\right\| \\
& \leq \omega\left(\left\|x_{0}\right\|+R\right)\left\|x_{i}-x_{i-1}\right\|,
\end{aligned}
$$


since $x_{i-1}+t\left(x_{i}-x_{i-1}\right) \in B\left(x_{0}, R\right)$ and $\omega$ is a non-decreasing function. Therefore

$$
\left\|x_{i+1}-x_{i}\right\| \leq \omega\left(\left\|x_{0}\right\|+R\right)\left\|x_{i}-x_{i-1}\right\|
$$

and, by lemma 2.1 ,

$$
\begin{aligned}
\left\|x_{i+1}-x_{0}\right\| & \leq\left\|x_{i+1}-x_{i}\right\|+\left\|x_{i}-x_{0}\right\| \\
& <\left[1+\sum_{j=1}^{i} \omega\left(\left\|x_{0}\right\|+R\right)^{j}\right]\left\|x_{1}-x_{0}\right\| \\
& \leq\left[1+\sum_{j=1}^{i} \omega\left(\left\|x_{0}\right\|+R\right)^{j}\right] \eta<R .
\end{aligned}
$$

Mathematical induction now completes the proof.

After that we can prove the semilocal convergence of method (1) when it is applied to operators satisfying all $\left(\mathbf{C}_{1}\right)-\left(\mathbf{C}_{4}\right)$.

Theorem 2.3. Let $X$ be a Banach space and $F: \Omega \subseteq X \rightarrow X$ a differenciable operator on an open convex domain $\Omega$. Assume all conditions $\left(\boldsymbol{C}_{1}\right)-\left(\boldsymbol{C}_{4}\right)$. Then iteration (1), starting at $x_{0}$, converges to a solution $x^{*} \in \overline{B\left(x_{0}, R\right)}$ of the equation $F(x)=0$.

Proof. From lemma 2.2, it follows that the sequence defined by (1) is a Cauchy one, since, for $k \geq 1$ and $n \geq 1$, we have

$$
\begin{aligned}
\left\|x_{n+k}-x_{n}\right\| & \leq\left\|x_{n+k}-x_{n+k-1}\right\|+\left\|x_{n+k-1}-x_{n+k-2}\right\|+\cdots+\left\|x_{n+1}-x_{n}\right\| \\
& \leq \sum_{i=n}^{n+k-1}\left(\omega\left(\left\|x_{0}\right\|+R\right)^{i-n+1}\right) \omega\left(\left\|x_{0}\right\|+R\right)^{n-1}\left\|x_{1}-x_{0}\right\| \\
& =\frac{1-\omega\left(\left\|x_{0}\right\|+R\right)^{k}}{1-\omega\left(\left\|x_{0}\right\|+R\right)} \omega\left(\left\|x_{0}\right\|+R\right)^{n}\left\|x_{1}-x_{0}\right\|
\end{aligned}
$$

and $\omega\left(\left\|x_{0}\right\|+R\right)<1$. In consequence, $\lim _{n} x_{n}=x^{*}$, and by letting $n \rightarrow \infty$ in (5) for $i=n$, we obtain $F\left(x^{*}\right)=0$ by the continuity of $F$ in $\overline{B\left(x_{0}, R\right)}$. 
Example. We now illustrate the previous analysis to solve (2) by applying method (1). Note that solving (2) is equivalent to solve $F(x)=0$, where $F: C[0,1] \rightarrow C[0,1]$ and

$$
[F(x)](s)=x(s)-s-\frac{1}{20} \int_{0}^{1} K(s, t) x(t)^{5} d t .
$$

Thus

$$
\left[F^{\prime}(x) y\right](s)=y(s)-\frac{1}{4} \int_{0}^{1} K(s, t) x(t)^{4} y(t) d t
$$

and $\omega(t)=t^{4} / 32$.

Firstly, we obtain conclusions about the existence and the location of a solution $x^{*}$ of (2), by means of Theorem 2.3. If we choose $x_{0}(s)=0$, then $\eta=1$, the equation appearing in $\left(\mathbf{C}_{3}\right)$ is reduced to $32-32 t+t^{5}=0$ and $R=1.03758 \ldots$ Consequently, by Theorem 2.3, equation (2) has the solution $x^{*}$ in

$$
\{u \in C[0,1] ;\|u\| \leq 1.03758 \ldots\} .
$$

After six iterations, the solution $x^{*}(s)=1.0012 s-0.00119048 s^{7}$ is approximated by Picard's method. See figure 1 .

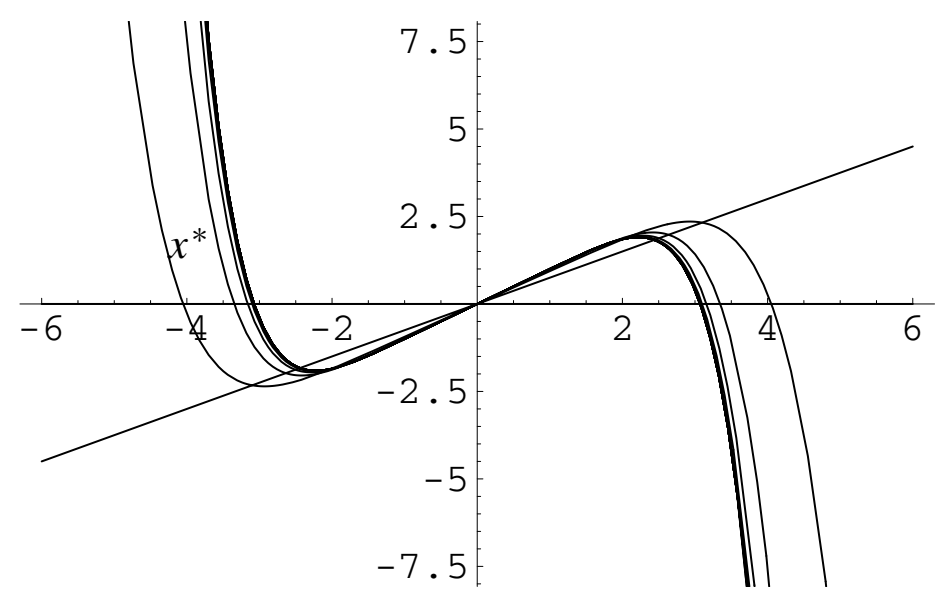

Figure 1 - Approximation of $x^{*}$ by Picard's iteration. 


\section{Application}

In this section we present an application where the analysis given in the previous section is applied. The convergence condition given in (4) is easy to apply to nonlinear integral equations of Fredholm type and second kind:

$$
x(s)=f(s)+\lambda \int_{a}^{b} K(s, t) H(x)(t) d t, \quad a \leq s \leq b,
$$

where $\lambda$ is a real number, the kernel $K(s, t)$ is a continuous function in $[a, b] \times$ $[a, b], H: C[a, b] \longrightarrow C[a, b]$ is a differentiable operator, and $f(s)$ is a given continuous function defined in $[a, b]$.

Note that solving (7) is equivalent to solve $F(x)=0$, where $F: C[a, b] \rightarrow$ $C[a, b]$ and

$$
[F(x)](s)=x(s)-f(s)-\lambda \int_{a}^{b} K(s, t) H(x)(t) d t .
$$

Thus

$$
\left[F^{\prime}(x) y\right](s)=y(s)-\lambda \int_{a}^{b} K(s, t) H^{\prime}(x) y(t) d t
$$

Therefore

$$
\left\|F^{\prime}(x)-I_{C[a, b]}\right\| \leq|\lambda| M\left\|H^{\prime}(x)\right\|,
$$

where $M=\max _{[a, b]} \int_{a}^{b}|K(s, t)| d t$. Then, by giving conditions for $\left\|H^{\prime}(x)\right\|$, condition (4) can be satisfied. Note that the space of continuous functions on the interval $[a, b]$ is equipped with the max-norm

$$
\|h\|=\max _{s \in[a, b]}|h(s)|, \quad h \in C[a, b] .
$$

Firstly, we consider the following usual condition:

$$
\left\|H^{\prime}(u)-H^{\prime}(v)\right\| \leq L\|u-v\|^{p}, \quad L \geq 0 \quad \text { and } \quad p \in[0,1],
$$

namely, $H^{\prime}$ is $(L, p)$-Hölder continuous (see [3], [4]). Observe that $H^{\prime}$ is Lipschitz continuous if $p=1$. For the former case, if $x_{0} \in C[0,1]$, we can consider

$$
\left\|H^{\prime}(x)\right\| \leq\left\|H^{\prime}\left(x_{0}\right)\right\|+L\left\|x-x_{0}\right\|^{p} .
$$


In this case,

$$
\left\|F^{\prime}(x)-I_{C[a, b]}\right\| \leq|\lambda| M \tilde{\omega}\left(\left\|x-x_{0}\right\|\right) \equiv \omega\left(\left\|x-x_{0}\right\|\right),
$$

where $\tilde{\omega}: \mathbb{R}_{+} \rightarrow \mathbb{R}_{+}$with $\tilde{\omega}(t)=N+L t^{p}$ and $N=\left\|H^{\prime}\left(x_{0}\right)\right\|$. This new condition makes a slight variation in the previous semilocal convergence result. From (5), we obtain

$$
\left\|F\left(x_{i}\right)\right\| \leq \omega(R)\left\|x_{i}-x_{i-1}\right\|
$$

since $x_{i-1}+t\left(x_{i}-x_{i-1}\right) \in B\left(x_{0}, R\right)$, and condition $\left(\mathbf{C}_{3}\right)$ is transformed in the following one: Suppose $R$ exists, the smallest positive root of the equation

$$
|\lambda| M L t^{1+p}-(1-|\lambda| M N) t+\eta=0 .
$$

We illustrate these facts with the following example (see [5]):

$$
x(s)=s+\lambda \int_{0}^{1} K(s, t) x(t)^{2} d t,
$$

where $K$ is the Green function given in (3). For this nonlinear integral equation, the operator $F$ is

$$
F(x)(s)=x(s)-s-\lambda \int_{0}^{1} K(s, t) x(t)^{2} d t
$$

and

$$
F^{\prime}(x) y(s)=y(s)-2 \lambda \int_{0}^{1} K(s, t) x(t) y(t) d t .
$$

Then, $p=1, H^{\prime}(x)$ is Lipschitz continuous,

$$
\left\|F^{\prime}(x)-I_{C[0,1]}\right\| \leq|\lambda|\left(N+2\left\|x-x_{0}\right\|\right) / 8
$$

and equation (8) is reduced to the following polynomial of second degree:

$$
\frac{|\lambda|}{4} t^{2}-\left(1-\frac{|\lambda|}{8} N\right) t+\eta=0
$$

where $\eta=\left\|x_{0}-s\right\|+|\lambda| M\left\|x_{0}\right\|^{2}$ and the corresponding semilocal convergence result is now. 
Corollary 3.1. Let $R$ be the smallest positive root of equation (10). Then the sequence defined in (1), starting at $x_{0}$, converges to a root of (9), which is in $\overline{B\left(x_{0}, R\right)} \subset \Omega$.

If we choose $x_{0}(s)=s$, then $N=2, \eta=|\lambda| / 8$ and equation (10) is

$$
\frac{|\lambda|}{4} t^{2}-\left(1-\frac{|\lambda|}{4}\right) t+\frac{|\lambda|}{8}=0,
$$

which has real solutions if $|\lambda| \in(0,4 \sqrt{2}-4)$. Taking $\lambda=1$, we obtain $R=\frac{3-\sqrt{7}}{2}$ and equation (9) with $\lambda=1$ has a solution in

$$
\left\{u \in C[0,1] ;\|u-1\| \leq \frac{3-\sqrt{7}}{2}\right\} .
$$

By iteration (1), starting at $x_{0}(s)=s$, the solution

$$
x^{*}(s)=1.10542-0.00184454 s^{2}-0.0202459 s^{3}-0.0833333 s^{4}
$$

is reached at the iteration 10 .

Secondly, we consider the more general situation

$$
\left\|H^{\prime}(x)\right\| \leq \tilde{\omega}(\|x\|)
$$

where $\tilde{\omega}: \mathbb{R}_{+} \rightarrow \mathbb{R}_{+}$is a non-decreasing function. In this case, $\omega(t)=$ $|\lambda| M \tilde{\omega}(t)$. We illustrate this situation with the following boundary value problem

$$
\left\{\begin{array}{l}
\frac{d^{2} x(s)}{d s^{2}}+\lambda \mathrm{e}^{x(s)}=0, \quad s \in[0,1], \\
x(0)=0=x(1),
\end{array}\right.
$$

which can be written in the form of the next nonlinear integral equation

$$
x(s)=\lambda \int_{0}^{1} K(s, t) \mathrm{e}^{x(t)} d t,
$$

where $K$ is the Green function given in (3). Moreover, solving (12) is equivalent to solve $F(x)=0$, where $F: C[0,1] \rightarrow C[0,1]$ and

$$
[F(x)](s)=x(s)-\lambda \int_{0}^{1} K(s, t) \mathrm{e}^{x(t)} d t .
$$


In addition, $\eta=\left\|x_{0}\right\|+|\lambda| \mathrm{e}^{\left\|x_{0}\right\|} / 8$, Picard's iteration is reduced to

$$
x_{n+1}(s)=\lambda \int_{0}^{1} K(s, t) \mathrm{e}^{x_{n}(t)} d t
$$

and the semilocal convergence result is now.

Corollary 3.2. Let $R$ be the smallest positive root of the equation

$$
\left(1-|\lambda| \mathrm{e}^{\left\|x_{0}\right\|+t} / 8\right) t-\left\|x_{0}\right\|-|\lambda| \mathrm{e}^{\left\|x_{0}\right\|} / 8=0
$$

Then the sequence defined in (1), starting at $x_{0}$, converges to a zero of (13), which is in $\overline{B\left(x_{0}, R\right)} \subset \Omega$.

If we choose $x_{0}(s)=1$, equation (14) is

$$
\left(1-|\lambda| \mathrm{e}^{1+t} / 8\right) t-1-|\lambda| \mathrm{e} / 8=0,
$$

so that, as we can see in figure 2 , the smaller the value $|\lambda|$ is, the smaller the value $R$ is. Taking $\lambda=1 / 10, R=1.15962 \ldots$ is obtained and problem (11) with $\lambda=1 / 10$ has a solution $x^{* *}$ in

$$
\{u \in C[0,1] ;\|u-1\| \leq 1.15962 \ldots\}
$$

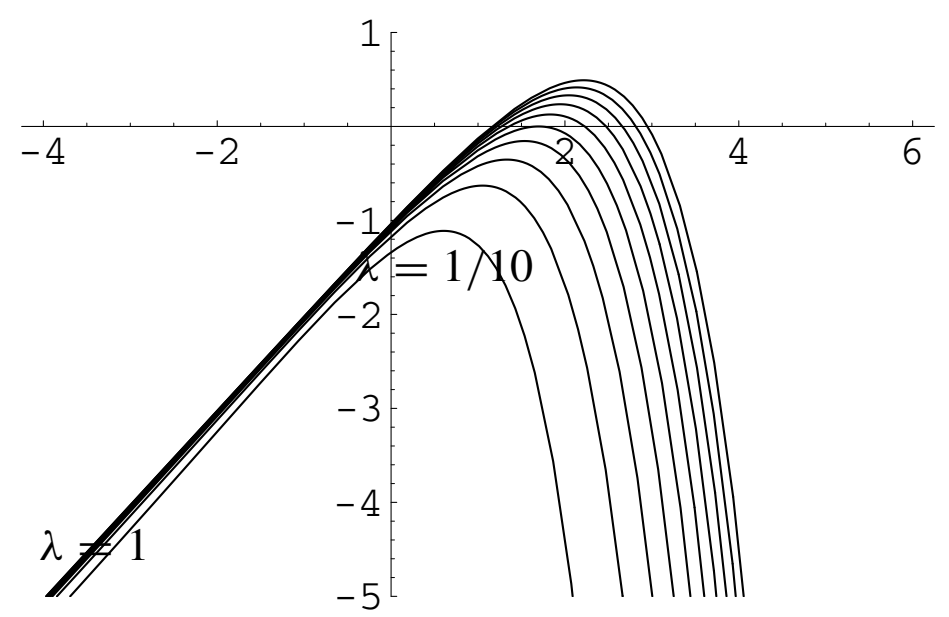

Figure $2-h_{1}(t)=\left(1-|\lambda| \mathrm{e}^{1+t} / 8\right) t-1-|\lambda| \mathrm{e} / 8$ for $\lambda=1 / n(n=1,2, \ldots, 10)$. 
The solution $x^{* *}(s)=0.0508548 s-0.0508548 s^{2}$ of $(11)$ with $\lambda=1 / 10$ is approximated by Picard's iteration in the sixth iteration.

\section{REFERENCES}

[1] M. Altman, Contractors and contractor directions theory and applications, Marcel Dekker, New York and Basel, (1977).

[2] I. K. Argyros, Newton-like methods under mild differentiability conditions with error analysis, Bull. Austral. Math. Soc., 37 (1) (1988), 131-147.

[3] I. K. Argyros, The Newton-Kantorovich method under mild differentiability conditions and the Pták error estimates, Monatsh. Math., 101 (1990), 175-193.

[4] I. K. Argyros, Remarks on the convergence of Newton's method under Hölder continuity conditions, Tamkang J. Math., 23 (4) (1992), 269-277.

[5] H. T. Davis, Introduction to Nonlinear Differential and Integral Equations, Dover Publications, New York, (1962).

[6] J. A. Ezquerro and M. A. Hernández, Generalized Differentiability Conditions for Newton's Method, IMA J. Numer. Anal., 22 (2002), 187-205.

[7] A. K. Kalinde, Solving some Nonlinear Equations by Successive Approximations, Int. J. Math. Math. Sci., 31 (6) (2002), 353-358.

[8] L. V. Kantorovich and G. P. Akilov, Functional analysis, Pergamon Press, Oxford, (1982).

[9] L. B. Rall, Computational Solution of Nonlinear Operator Equations, John Wiley \& Sons, New York, (1979).

[10] Song-bai Sheng and Hui-fu Xu, Picard Iteration for Nonsmooth Equations, J. Comput. Math., 19 (6) (2001), 583-590. 